\title{
Interleukin-17a as a predictor of occurrence of sepsis in polytrauma patients: a prospective observational study
}

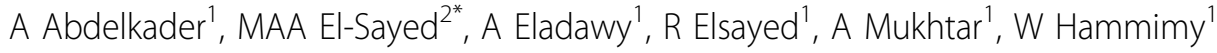 \\ From ESICM LIVES 2015 \\ Berlin, Germany. 3-7 October 2015
}

\section{Introduction}

One of the most serious complications of major trauma is the sequential dysfunction of vital organs, mostly associated with posttraumatic sepsis. IL-17 has been linked to the severity of inflammation in tissues. It initiates the production of other pro-inflammatory mediators resulting in an influx of neutrophils.

\section{Objectives}

To investigate whether the level of IL17A at the day of admission could predict the occurrence of sepsis in polytrauma patients or not.

\section{Methods}

A prospective cohort study in polytrauma patients. 100 consecutive adult polytrauma patients were included. Serum level of IL-17A was measured at the day of admission to the intensive care unit (ICU). Patients were screened for the development of sepsis. Other data collection included; demographic data Abbreviated Injury Scale (AIS), APACHE II score, acute kidney injury assessed by RIFLE criteria, acute respiratory distress syndrome (ARDS), duration of mechanical ventilation, ICU length of stay \& 28-day mortality.

\section{Results}

Out of 100 patients 47 (47\%) developed sepsis. Serum level of IL-17A was significantly higher in the group of patients who developed sepsis compared to the non-septic group (p-value 0.004). The optimum cut-off value of serum IL-17A for sepsis prediction in polytrauma patients was $\geq 53.8 \mathrm{pg} / \mathrm{ml}$. This cut-off value had a sensitivity of
$60.7 \%$ \& a specificity of $76.4 \%$, area under the curve (AUC) was 0.687 (95\% CI 0.573-0.802), (p value 0.004), as shown in Figure 1. High serum level of IL17-A was associated with increased 28 days ICU mortality as shown in Figure 2 ( $\mathrm{p}$ value of 0.06 ).

Neither ARDS nor Multiple Organ Dysfunction Score (MODS) was associated with increased IL-17A serum level.

\section{Conclusions}

IL-17A is a potential predictor of sepsis occurrence in patients with polytrauma.

\section{Authors' details}

${ }^{1}$ Cairo University, Cairo, Egypt. ${ }^{2}$ Cairo University, Anesthesia, Cairo, Egypt.

Published: 1 October 2015

Reference

1. Nakada TA, Russell JA, Boyd JH, et al: IL 17A genetic variation is associated with altered susceptibility to Gram-positive infection and mortality of severe sepsis. Crit Care 2011, 15:R254.

doi:10.1186/2197-425X-3-S1-A790

Cite this article as: Abdelkader et al:: Interleukin-17a as a predictor of occurrence of sepsis in polytrauma patients: a prospective observational study. Intensive Care Medicine Experimental 2015 3(Suppl 1):A790. 